Ann. Génét. Sél. anim., 1976, 8 (2), I73-180.

\title{
GROUPES SANGUINS ET MALADIE DE MAREK CHEZ LA POULE : RÉSISTANCE D'UN GÉNOTYPE HÊTÉROZYGOTE
}

\author{
G. BENNEJEAN, P. MÉRAT*, A. PERRAMON*, \\ M. GUITTET et J.-P. PICAULT \\ Station expérimentale d'Aviculture, \\ Laboratoire de Recherches vétérinaires, \\ 22440 Ploufragan, Saint-Brieuc \\ * Laboratoire de Génétique factorielle, \\ Centre national de Recherches zootechniques, I. N.R.A., \\ 78350 Jouy en Josas
}

\begin{abstract}
RÉSUMÉ
Des comparaisons entre sexes, entre familles de pères et entre génotypes à trois loci codant des antigènes de la membrane érythrocytaire ont été faites relativement à la résistance (lésions macroscopiques) au virus de la maladie de Marek. Ce dernier était inoculé aux poussins après l'éclosion deux années de suite dans une population expérimentale de race Rhode-Island. Il n'apparaît pas de différences entre sexes, et seulement une année sur deux entre familles. Un locus pour des antigènes membranaires $(I)$ est peut-être neutre dans nos conditions vis-à-vis de la sensibilité au virus Marek, une différence significative en 1974 à l'avantage de l'ensemble des hétérozygotes restant à confirmer. Une différence observée en I974 au locus $I I I$ n'est pas retrouvée en 1975 . Par contre, au locus $I I$, un génotype hétérozygote présente les deux années une résistance bien meilleure à ce virus que l'ensemble des autres génotypes.
\end{abstract}

\section{INTRODUCTION}

Plusieurs chercheurs ont fait état de différences, parfois importantes, de sensibilité à la maladie de Marek associées à des génotypes de groupe sanguin chez le Poulet (par ex. HANSEN et al., I967 ; BREWER et al., I969 ; JOHNSON, I973 ; BRILES, I974I975; BRILES et SCHELIING, I975 ; Johnson et al., I975). A l'exception de BRILES (I974) et de BRILES et SChELLING (I975), il s'agit toujours de comparaisons entre allèles du locus $B$. Ces comparaisons concernent le plus souvent des lignées de race Leghorn blanche. Sauf dans un cas (Jounson et al., I975) elles ne paraissent pas 
planifiées de façon à faire ressortir des phénomènes éventuels d'hétérosis. Il paraît donc intéressant de mentionner nos résultats qui précisément montrent un tel phénomène dans une population expérimentale de race Rhode-Island rouge.

\section{MATÉRIEL ET MÉTHODES}

Une première éclosion avait lieu le 3-4-I974 à Jouy-en-Josas. Les poussins, au nombre total de 267 , étaient issus de familles présentant une ségrégation à trois loci distincts de groupe sanguin, antérieurement décrits (PERRAMon et MÉrat, 1968).

Ils étaient envoyés dès après l'éclosion au Laboratoire de Recherches vétérinaires à Saint-BrieucPloufragan où ils ont été infectés à l'âge de 4 jours avec $0,2 \mathrm{ml}$ de sang total, citraté au quart, provenant de poulets présentant des lésions macroscopiques de la maladie de Marek. Les poulets donneurs avaient eux-mêmes été infectés par la même voie avec 250 unités formant plaques de la souche Ploufragan de virus de la maladie de Marek cultivé sur fibroblastes de poulet et conservée à $-196^{\circ} \mathrm{C}$.

Le génotype sanguin était déterminé individuellement à partir d'un volume de I cc de sang prélevé en tube citraté, à l'âge de 4 semaines, puis envoyé au laboratoire de génétique factorielle à Jouy en Josas.

Pendant toute la période d'observation d'une durée de 17 semaines, les animaux ont été répartis de façon aléatoire en cages dans une animalerie équipée d'un dispositif de filtration absolue de l'air et d'un sas de douche pour le personnel. L'aliment après avoir subi un traitement thermique était mis en sac plastique désinfecté par les vapeurs de formol avant l'entrée dans l'animalerie.

Chaque jour les animaux morts ont été autopsiés et les lésions macroscopiques viscérales ou nerveuses de la maladie de Marek répertoriées. En fin d'expérience, tous les animaux survivants ont été sacrifiés et autopsiés.

En raison du nombre peu élevé d'animaux porteurs de lésions à l'abattage, nous avons regroupé en une seule catégorie tous les porteurs de lésions vivants ou morts.

Au total le nombre de poussins morts s'élevait à $90(33,7$ p. roo) dont 67 (soit 25, I p. 100) avec des lésions apparentes caractéristiques de la maladie de Marek.

Les trois loci sont désignés par $I, I I$ et $I I I$. Le locus $I$ comporte dans notre échantillon trois allèles identifiés appelés $1,7,8$; deux allèles (2 et 4 ) sont reconnus au locus $I I\left({ }^{1}\right)$; au locus $I I I$, un seul allèle (6) est identifié $\left({ }^{2}\right)$. A chacun des loci, l'absence des allèles connus est désignée par o.

En 1975, une comparaison était faite dans les mêmes conditions (inoculation, diagnostic de l'antigène, âge d'abattage...) sur 224 poussins d'origine identique éclos le 2-04.

Dans cette seconde expérience une mortalité importante apparue dès la mise en place des animaux a persisté pendant les quatre premières semaines. Bien que l'absence de lésions caractéristiques ne permette pas d'en attribuer la cause à la maladie de Marek, il est possible d'affirmer à la suite de différentes observations, que la mortalité de type non spécifique est toujours beaucoup plus élevée dans les lots inoculés que dans les lots témoins.

En conséquence, la mortalité globale attribuable à la maladie de Marek ne s'élevait qu'à 12,5 p. Ioo et le pourcentage total d'animaux porteurs de lésions macroscopiques à I 5,6 p. I00. Nous avons donc préféré considérer séparément chaque essai, les tests de signification étant faits dans les deux cas sur le pourcentage de lésions (morts et vivants groupés).

Dans tous les cas, les tests de $\chi^{2}$ sont faits compte tenu de la correction de Yates.

\section{RÉSULTATS}

\section{I. - Test de 1974}

En détaillant trois catégories (vivants sans lésions, porteurs de lésions, morts sans lésions) les résultats sont présentés par sexe (tabl. I).

( $\left.{ }^{1}\right)$ L'allèle 2 n'était pas identifié lors de notre publication initiale (PERramon et MÉrat, 1968).

(2) L'allèle 5 mentionné dans la publication initiale n'est pas identifié ici. 
TABLEAU I

Comparaison entre sexes pour la sensibilité à la maladie de Marek (1974)

Comparison between sexes for susceptibility to Marek's disease (1974)

\begin{tabular}{|c|c|c|c|c|c|c|c|}
\hline \multirow[t]{2}{*}{$\begin{array}{l}\text { Sexe } \\
(\operatorname{Sex})\end{array}$} & \multicolumn{2}{|c|}{$\begin{array}{l}\text { Survivants sans lésions } \\
\text { (Survivors without lesions) }\end{array}$} & \multicolumn{2}{|c|}{$\begin{array}{l}\text { Porteurs de lésions } \\
\text { (vivants ou morts) } \\
\text { (Carriers of lesions } \\
\text { (alive or dead)) }\end{array}$} & \multicolumn{2}{|c|}{$\begin{array}{c}\text { Morts } \\
\text { sans lésions visibles } \\
\text { (Dead } \\
\text { without visible lesions) }\end{array}$} & \multirow{2}{*}{$\begin{array}{c}\text { Total } \\
\text { (Total) } \\
\mathrm{N}\end{array}$} \\
\hline & $N$ & $\%$ & $\mathrm{~N}$ & $\%$ & $\mathbf{N}$ & $\%$ & \\
\hline 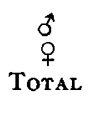 & $\begin{array}{r}96 \\
81 \\
177\end{array}$ & $\begin{array}{l}65,3 \\
67,5 \\
66,3\end{array}$ & $\begin{array}{l}36 \\
31 \\
67\end{array}$ & $\begin{array}{l}24,5 \\
25,8 \\
25,1\end{array}$ & $\begin{array}{r}15 \\
8 \\
23\end{array}$ & $\begin{array}{r}10,2 \\
6,7 \\
8,6\end{array}$ & $\begin{array}{l}147 \\
120 \\
267\end{array}$ \\
\hline
\end{tabular}

TABLEAU 2

Comparaison entre génotypes au locus I pour la sensibilité à la maladie de Marek (1974)

Comparison between genotypes at locus I for susceptibility to Marek's disease (1974)

\begin{tabular}{|c|c|c|c|c|c|c|c|c|}
\hline & \multicolumn{8}{|c|}{$\begin{array}{l}\text { Phénotypes comparés } \\
\text { (Compared phenotypes) }\end{array}$} \\
\hline & 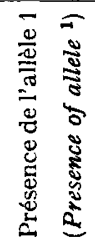 & 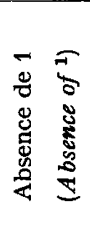 & 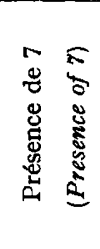 & 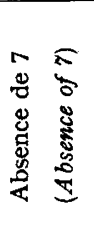 & 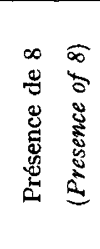 & 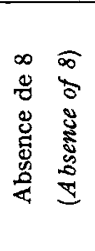 & 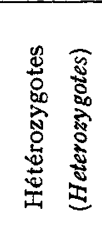 & 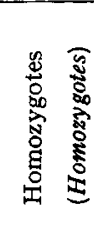 \\
\hline $\begin{array}{c}\text { Vivants sans lésions } \\
\text { (Survivors } \\
\text { without lesions) }\end{array}$ & 92 & 75 & 81 & 99 & 61 & 102 & $77^{\prime}$ & 89 \\
\hline $\begin{array}{l}\text { Porteurs de lésions } \\
(\mathrm{v} . \text { ou m.) } \\
(\text { Carriers of lesions } \\
(\text { a. or } d .))\end{array}$ & 20 & 29 & 20 & 44 & 20 & 39 & 17 & 42 \\
\hline $\begin{array}{l}\% \text { lésions } \\
(\% \text { lesions })\end{array}$ & 22,0 & 27,9 & 19,8 & 30,8 & 24,7 & 27,7 & 18,7 & 32,1 \\
\hline & $\chi^{2}=0$ & N. S.) & $\chi^{2}=3$ & (N. S.) & $\chi^{2}=$ & (N. S.) & $\begin{array}{l}\chi^{2} \\
(p\end{array}$ & $\begin{array}{l}4,28 \\
0,05)\end{array}$ \\
\hline
\end{tabular}


Pour simplifier, nous omettons dans tous les tests, ainsi que dans les tableaux suivants, la catégorie des morts sans lésions, incertaine quant à sa signification vis-àvis de la maladie de Marek et relativement peu nombreuse ( $\left.{ }^{1}\right)$. Le $\chi^{2}$ de contingence entre sexes ne comporte alors qu'un degré de liberté ; sa valeur est non significative. L'âge moyen de mortalité calculé par ailleurs ne diffère pas non plus suivant le sexe (respectivement II3 et II9 jours pour les poussins $\delta \hat{\jmath}$ et $ㅇ ㅜ ; t=0,93$, N.S.).

En fonction de ce résultat, toutes les comparaisons, entre génotypes et entre familles, sont faites en groupant les sexes. Chaque comparaison entre génotypes à un locus est limitée aux familles de même père comportant une ségrégation pour les génotypes étudiés.

Le tableau 2 donne les nombres et pourcentages de "vivants sans lésions " et de " porteurs de lésions" (morts ou survivants) pour les génotypes correspondant au locus $I$. En raison du nombre d'allèles et des effectifs limités, les comparaisons présentées concernent seulement, pour ce locus, la présence ou l'absence de chaque allèle; nous y ajoutons une comparaison globale " hétérozygotes-homozygotes ", tous les génotypes étant regroupés.

Le tableau 3 présente la seule comparaison (présence ou absence de l'allèle 6) obtenue pour le locus $I I I$.

TABLEAU 3

Comparaison entre génotypes au locus III pour la sensibilité à la maladie de Marek (1974)

Comparison between genotypes at locus III

for susceptibility to Marek's disease (1974)

\begin{tabular}{|c|c|c|}
\hline & \multicolumn{2}{|c|}{$\begin{array}{l}\text { Phénotypes } \\
\text { (Phenotypes) }\end{array}$} \\
\hline & $\begin{array}{l}\text { Présence de } 6 \\
(\text { Presence of } 6)\end{array}$ & $\begin{array}{l}\text { Absence de } 6 \\
(A \text { bsence of } 6)\end{array}$ \\
\hline $\begin{array}{l}\text { Vivants sans lésions } \\
\text { (Survivors without lesions) }\end{array}$ & 87 & 102 \\
\hline $\begin{array}{l}\text { Porteurs de lésions (v. ou m.) } \\
\text { (Carriers of lesions }(a . \text { or } d .))\end{array}$ & 41 & 20 \\
\hline \multirow[t]{2}{*}{$\begin{array}{c}\% \text { lésions } \\
(\% \text { lesions })\end{array}$} & 32,0 & 16,4 \\
\hline & \multicolumn{2}{|c|}{$\chi^{2}=7,50(\mathrm{P}<0,01)$} \\
\hline
\end{tabular}

Le tableau 4 contient 2 comparaisons entre génotypes incluant les allèles 2 et 4 (locus $I I$ ), faites dans des groupes de familles différents, une troisième colonne contenant la comparaison globale entre le génotype $2 / 4$ et les autres génotypes.

(1) Nous avons d'ailleurs vérifié qu'en ajoutant cette catégorie à celle des morts porteurs de lésions, aucun des résultats ne serait se nsiblement modifié. 
Une comparaison entre familles de pères a été faite séparément, mais en la restreignant aux animaux de génotype autre que $2 / 4$ (locus $I I$ ), en fonction des résultats précédents. Le $\chi^{2}$ pour 8 degrés de liberté est égal à $8,3 \mathrm{I}$, non significatif.

Par ailleurs, le détail de la répartition des types de lésions observées (neurales, viscérales ou les deux ensemble) ne fait apparaître de divergences sensibles entre génotypes pour aucun locus, et nous l'omettons ici. Il en est de même de 1'âge moyen de mortalité, y compris entre génotypes au locus $I I$ (respectivement III et II6 jours pour le génotype $2 / 4$ et l'ensemble des autres).

\section{TABLEAU 4}

Comparaison entre génotypes au locus II pour la sensibilité à la maladie de Marek (1974)

Comparison between genotypes at locus II for susceptibility to Marek's disease (1974)

\begin{tabular}{|c|c|c|c|c|c|c|c|}
\hline & \multicolumn{7}{|c|}{$\begin{array}{l}\text { Comparaison } \\
\text { (Comparison) }\end{array}$} \\
\hline & $\begin{array}{c}4 / 4 \\
\text { ou } 4 / 0\end{array}$ & $\begin{array}{l}2 / 2 \\
\text { ou } 2 / 0\end{array}$ & $2 / t$ & $\begin{array}{l}2 / 2 \\
\text { ou } 2 / 0\end{array}$ & $-2 / k$ & $2 / 4$ & $\begin{array}{l}\text { Autres } \\
\text { géno- } \\
\text { types }\end{array}$ \\
\hline $\begin{array}{c}\text { Vivants sans lésions } \\
\text { (Survivors without lesions) }\end{array}$ & 35 & 43 & 29 & 20 & $1^{\prime} t$ & 43 & 98 \\
\hline $\begin{array}{l}\text { Porteurs de lesions (v. ou m.) } \\
\text { (Carriers of lesions (a. or } d .) \text { ) }\end{array}$ & 20 & 19 & 1 & Ј & 1 & $\because$ & 41 \\
\hline $\begin{array}{l}\% \text { lésions } \\
\% \text { (lesions) }\end{array}$ & 36,4 & 30,6 & 3,3 & $25,1)$ & 6,7 & $4, t^{\prime}$ & 31,0 \\
\hline $\begin{array}{l}\text { Comparaison entre génotype } 2 / 4 \\
\text { et autres génotypes }\end{array}$ & \multicolumn{3}{|c|}{$\chi^{2}=9,38(P<0,005)$} & & & \multicolumn{2}{|c|}{ 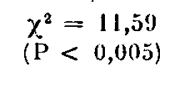 } \\
\hline
\end{tabular}

\section{2. - Test de 1975}

Nous n'avons pas jugé utile de présenter tous les détails des données de I975. Elles confirment celles de 1974 en ce qui concerne 1'absence de différences entre sexes. Au locus $I$, seuls quatre groupes (I/I ou $\mathrm{I} / \mathrm{O}, \mathrm{I} / 7, \mathrm{I} / 8,7 / 8)$ pouvaient être comparés eu égard aux effectifs disponibles. Le $\chi^{2}$ correspondant est non significatif $(2,04$ pour 3 d.1.). Au locus $I I I$, la comparaison entre présence et absence de l'allèle 6 (respecti-

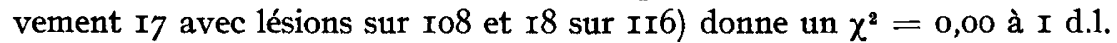

Quant au locus $I I$, la comparaison entre le génotype $2 / 4$ et les autres catégories (après vérification de l'absence de différence appréciable entre ces dernières) figure au tableau 5. Comme lors du test de 1974, la comparaison est limitée aux familles de même père contenant les deux catégories.

Enfin, on note cette fois des différences appréciables entre les ro familles de même père présentes $\left(\chi^{2}\right.$ à $\left.9 \mathrm{~d} .1 .=33,60, \mathrm{P}<0,00 \mathrm{r}\right)$. Malgré les effectifs calculés relativement faibles dans certaines familles, la valeur du $\chi^{2}$ est suffisante pour montrer l'existence de différences familiales. 
TABLEAU 5

Comparaison entre génotypes au locus II pour la sensibilité à la maladie de Marek (1975)

Comparison between genotypes at locus II for susceptibility to Marek's disease (1975)

\begin{tabular}{c|c|c}
\hline \hline & \multicolumn{2}{|c}{$\begin{array}{c}\text { Comparaison } \\
\text { (Comparison) }\end{array}$} \\
\hline $\begin{array}{c}\text { Vivants sans lésions } \\
\text { (Survivors without lesions) }\end{array}$ & $2 /{ }^{\prime}$ & $\begin{array}{c}\text { Autres génotypes } \\
\text { (Other genotypes) }\end{array}$ \\
\hline $\begin{array}{c}\text { Porteurs de lésions (v. ou m.) } \\
\text { (Alive or dead with lesions) }\end{array}$ & 46 & $10^{\prime}$ \\
\hline $\begin{array}{c}\% \text { lésions } \\
(\% \text { lesions })\end{array}$ & -4 & 20 \\
\hline
\end{tabular}

\section{DISCUSSION}

Au locus $I$, en $197+$ comme en I975, la présence d'aucun allèle ne s'accompagne d'une différence significative (tabl. 2), non plus que certaines comparaisons plus détaillées qui étaient possibles (entre génotypes $\mathrm{I} / \mathrm{I}, \mathrm{I} / \mathrm{O}, \mathrm{I} / 7, \mathrm{I} / 8$ ). Les données de I974 suggèrent un avantage des génotypes hétérozygotes au total, celles de I975 ne paraissant pas concluantes à cet égard.

Au locus $I I I$ en I974, l'allèle 6 s'accompagne d'un pourcentage plus élevé de lésions et de mortalité (tabl. 3) mais l'absence de différence décelable l'année suivante ne permet pas une conclusion définitive.

Concernant le locus $I I$, il apparaît clairement en I974 que l'hétérozygote $2 / 4$ a une mortalité très inférieure à celle des autres catégories (tabl. 4), celles-ci ne présentant pas d'écarts importants entre elles. L'année suivante, la comparaison correspondante n'est pas significative au seuil de $5 \mathrm{p}$. Ioo (tabl. 5 ), mais le résultat est de même sens. Nos observations semblent donc indiquer une composante hétérotique monogénique au niveau d'un locus codant pour des caractères antigéniques de membrane érythrocytaire.

La plupart de nos comparaisons entre génotypes étaient faites intra-mères, et en ne conservant que de telles comparaisons, l'effet observé subsiste : restreinte aux descendances de mères contenant à la fois le génotype $2 / 4$ et d'autres génotypes, la comparaison du pourcentage de lésions entre ces deux groupes fait apparaître 2 porteurs de lésions sur 35 pour le premier, I8 sur 49 pour le second $\left(\chi^{2}=9\right.$, I8; $\mathrm{P}<0,005$. 
Par contre, une comparaison du même pourcentage entre enfants de génotype autre que $2 / 4$ descendant respectivement de mères $2 / 4$ ou de mères d'un génotype différent fait apparaître 22 porteurs de lésions sur 82 dans le premier cas et 33 sur 87 dans le second $\left(\chi^{2}=2,37, \mathrm{~N}\right.$. S.). On ne peut donc en fin de compte attribuer l'effet associé au locus $I I$ à un taux différent d'anticorps d'origine maternelle.

Nous n'avons pas jusqu'ici déterminé si le locus en cause est identique au locus $B$ des lignées de Leghorns sur lesquelles porte la plupart des études. Néanmoins BRILES et SCHELIING (I975) observent un effet semblable associé à plusieurs loci membranaires et de plus BRILES (I974-I975) compare des allèles au locus $B$ ségrégant chez des animaux issus du croisement de lignées différentes.

Chez la Souris des études expérimentales concernant la résistance à des infections virales fréquentes dans cette espèce, telles la chorioméningite lymphocytaire et l'ectromélie, mettent en évidence la supériorité des hétérozygotes au locus d'histocompatibilité H-2 (DOHERTY et ZINKERNAGEL, I975).

Le locus $B$ chez la Poule correspond lui aussi à un gène majeur d'histocompatibilité (par ex. Schierman et NoRDSKog, I96r ; CriTtenden et al., I964). Nous nous proposons d'étudier cet aspect chez nos souches afin de mieux cerner l'identification des facteurs antigéniques membranaires étudiés.

Concernant la possibilité d'existence d'un mécanisme hétérotique monogénique pour la résistance à la maladie de Marek, on peut signaler que tout récemment WErss (I975), dans une étude sur les composants membranaires de cellules d'embryon de poulet qui interviennent en tant que récepteurs pour certains virus tumorigènes à ARN, propose des modèles dans lesquels seul l'hétérozygote à un locus (bien que dans ce cas les allèles présents disposeraient de propriétés pléiotropiques), serait capable de bloquer le récepteur et d'empêcher l'infection virale.

Reçu pour publication en mars $19 \pi 6$.

\title{
REMERCIEMENT'S
}

Nous remercions les professeurs F. B. Hutt et R. K. ColE, Cornell University, et le docteur L. Cauchy, Station de Pathologie aviaive, I.N.R.A., Nouzilly, pour leurs très utiles critiques et remarques.

\section{SUMMARY}

\author{
BLOOD TYPES AND MAREK'S DISEASE IN THE FOWL :
} RESISTANCE OF A HETEROZYGOUS GENOTYPE

Comparisons were made for resistance to Marek's disease virus (macroscopic lesions) in an experimental strain of Rhode-Island Reds, between sexes, between sire families and between genotypes at three loci responsible for erythrocyte antigens. On two consecutive years, a preparation of the virus was inoculated after hatching. There was no difference between sexes, and only in one of the two years between families. One locus for erythrocyte antigens $(I)$ may be neutral for susceptibility to Marek's virus in our conditions, a significant difference in I974 at the advantage of heterozygotes on the whole remaining to be confirmed. At locus $I I I$, a difference found in $\mathbf{1 9 7 4}$ is not observed again in 1975. On the contrary, at locus $I I$, a heterozygous genotype shows on both years a much better resistance to this virus than the other genotypes altogether. 


\section{RÉFÉRENCES BIBL,IOGRAPHIQUES}

Briles IV. E., r974. Association between the B and R blood group loci and resistance to certain oncogenic viruses in chickens. C. R. 1er Congrès génél. appl. à la prod. anim., 1, 299-306.

Briles W. E., I975. Relative influence of B isoalleles from lines N and P on Marek's mortality. Poult. Sci., 54, I738-1739 (abstr.).

Briles W. E., Schelling E. P., 1975. Relative influence of marked chromosome segments on chick livability following J. M. Marek's challenge. Poult. Sci., 54, 1738 (abstr.).

Brewer R. N., Moore C. H., Johnson L. W., 1969. Effects of blood group antigens on resistance to Marek's disease. Poult. Sci., 48, r970 (abstr.).

Crittenden L. B., Johnson L. W., Okazaki W., i964. Histocompatibility and erythrocyte antigen variability within highly inbred lines of white Leghorns. Transplantation, 2, 362-374.

Doherty P. C., ZiNkERNAGel R. M., I975. Enhanced immunological surveillance in mice heterozygous at the H-2 gene complex. Nature, 235, 50-52.

Hansen M. P., Van Zandt J. N., LAw G. R. J., I967. Differences in susceptibility to Marek's Disease in chickens carrying two different B locus blood group alleles. Poult. Sci., 46, I268 (abstr.).

Johnson L. W., I973. Variation of life span in an inbred line caused by B blood antigen alleles. Poult. Sci., 52, 2045 (abstr.).

Johnson L. W., Brewer R. N., Moore C. H., i975. B blood antigen alleles affecting resistance and susceptibility to gross lesions of Marek's disease in primary broiler breeder lines. Poult. Sci., 54, 1779 (abstr.).

Pazderka F., Longenecker B. M., Law G. R J., Stone H. A., Ruth R. F., I975. Histocompatibility of chicken populations selected for resistance to Marek's Disease. Immunogenetics, 2, 93-Ioo.

Perramon A., Mérat P., rg68. Étude des groupes sanguins dans deux populations aviaires I. Transmission héréditaire des facteurs antigéniques. Ann. Biol. anim. bioch. biophys., 8, 485-50o.

Schierman L. W., Nordskog A. W., I961. Relationship of blood type to histocompatibility in chickens. Science, 134, 1008-1009.

Weiss R. Meeting "Cell Surface Discussion group " December 1975. Warwick. Nature, 247, 429-30. 